\title{
Hospital Management of Diabetes
}

\author{
Etie S. Moghissi, MD, FACP, FACE ${ }^{\mathrm{a}, *}$, \\ Irl B. Hirsch, MD ${ }^{\mathrm{b}, \mathrm{c}, *}$ \\ anpatient Diabetes and Metabolic Control Task Force, \\ American Association of Clinical Endocrinologists, 501 East Hardy Street, \\ Suite 110, Inglewood, CA 90301, USA \\ ${ }^{\mathrm{b}}$ Division of Metabolism, Endocrinology, and Nutrition, Department of Medicine, \\ University of Washington Medical Center, 1959 NE Pacific Street, \\ Box 356176, Seattle, WA 98195, USA \\ ${ }^{\mathrm{c}}$ Diabetes Care Center, University of Washington Medical Center, \\ Seattle, WA, USA
}

Diabetes remains a major cause of death and disability and is a growing global concern. The age-adjusted prevalence of type 2 diabetes in the United States increased by more than 55\% from 1990 to 2000 [1]. It is estimated that one out of three individuals born in 2000 will develop diabetes in their lifetime [2].

Chronic complications of diabetes, especially cardiovascular disease, result in hospitalization in many patients with diabetes. In 2001, more than 4.6 million hospitalizations were associated with diabetes, accounting for nearly 17 million hospital days at a cost of over \$40 billion [3].

Mounting observational and interventional evidence consistently indicates that hyperglycemia in the hospital setting is associated with increased mortality and morbidity and that meticulous glycemic control can improve clinical outcomes [4-7]. The purpose of this article is to review the evidence, discuss the importance of striving for good glycemic control in the hospital setting, and emphasize the need for additional outcome research studies to further examine the currently recommended in-hospital glycemic guidelines.

Dr. Morghissi is the former Vice-Chair of the Statement on Inpatient Diabetes and Metabolic Control writing panel of the American Association of Clinical Endocrinologists.

* Corresponding authors. Division of Metabolism, Endocrinology, and Nutrition, Department of Medicine, University of Washington Medical Center, 1959 NE Pacific Street, Box 356176, Seattle, WA 98195 (I.B. Hirsch).

E-mail addresses: emoghissi@pol.net (E.S. Moghissi); ihirsch@u.washington.edu (I.B. Hirsch). 
The article concludes with a discussion of strategies for achieving tight glycemic targets.

\section{Hyperglycemia in the hospital setting}

Hyperglycemia is a frequent finding in hospitalized patients. With the escalating incidence of diabetes in the United States, the number of patients with diabetes who require hospitalization has also increased. Between 1980 and 2001, the age-adjusted hospital discharge rate for diabetes as any-listed diagnosis in the general population increased over $49 \%$ from 109.0 to 162.4 per 10,000 in the general population [8]. A rapidly growing body of evidence clearly suggests that meticulous glycemic control improves clinical outcomes, including reduced mortality and infection rates [4-7]; however, until recently there were no established guidelines or standards for management of diabetes in the hospital setting.

In a recent consensus conference cosponsored by the American Association of Clinical Endocrinologists, American College of Endocrinology, American Diabetes Association, Endocrine Society, American Association of Diabetes Educators, American Heart Association, American Society of Anesthesiologists, Society of Critical Care Medicine, Society of Hospital Medicine, and Society of Thoracic Surgeons, a position statement was developed by the American College of Endocrinology with recommendations for glycemic targets in the hospital setting [9]. During the 2-day conference, experts reviewed extensive data and deliberated regarding the most appropriate targets for glycemic control in the hospital setting [9]. The upper limits of normal for glycemic targets are shown in Box 1.

\section{Role of intravenous insulin therapy in critically ill patients}

Hyperglycemia is very common in critically ill patients in the intensive care unit (ICU), even among patients with no prior history of diabetes. This

\section{Box 1. Upper limits for glycemic targets}

Intensive care unit

$110 \mathrm{mg} / \mathrm{dL}(6.1 \mathrm{mmol} / \mathrm{L})$

Noncritical care units

Preprandial

$110 \mathrm{mg} / \mathrm{dL}(6.1 \mathrm{mmol} / \mathrm{L})$

Maximal glucose

$180 \mathrm{mg} / \mathrm{dL}(10.0 \mathrm{mmol} / \mathrm{L})$

Data from American College of Endocrinology. Position statement on inpatient diabetes and metabolic control. Endocr Pract 2004;10:77-82. 
"stress hyperglycemia" is believed to be due to insulin resistance in the liver and the skeletal muscle. Until recently, it was felt that mild to moderate degrees of hyperglycemia was inconsequential or perhaps even adaptive to ensure there was enough substrate to provide enough energy.

Van den Berghe and colleagues [5] challenged this notion in a prospective, randomized control study. They studied 1548 mechanically ventilated adults who were admitted to a surgical ICU. These patients were randomized to receive either intensive insulin therapy with target blood glucose of 80 to 110 $\mathrm{mg} / \mathrm{dL}$ or conventional therapy to maintain blood glucose between 180 and $200 \mathrm{mg} / \mathrm{dL}$; insulin infusion was initiated in the conventional group if blood glucose exceeded $215 \mathrm{mg} / \mathrm{dL}$. The primary outcome measure was all-cause mortality in the ICU. Secondary outcome measures included in-hospital mortality, duration of ICU stay, and need for ICU ventilator support for more than 14 days.

The intensive insulin therapy group showed a significant reduction in ICU mortality compared with the conventional therapy group, $4.6 \%$ and $8 \%$ respectively $(P<0.04)$. The mortality reduction was only evident in patients who stayed in the ICU more than 5 days, $10.6 \%$ versus $20.2 \%$ $(P=0.005)$. In addition, the intensive therapy group had significant reductions in in-hospital mortality $(-34 \%)$, sepsis $(-46 \%)$, acute renal failure requiring dialysis or hemofiltration $(-41 \%)$, rate of transfusion $(-50 \%)$, and polyneuropathy $(-44 \%)$.

\section{Impact of hyperglycemia and intravenous insulin infusion on the outcome of myocardial infarction}

More than $80 \%$ of deaths associated with diabetes are from cardiovascular disease, of which $75 \%$ are a result of coronary artery disease [10]. Although the mortality from coronary artery disease has declined in the general population, this decline has not been as significant in individuals with diabetes. Insulin resistance and abnormal glucose tolerance is even more common in patients with acute coronary syndrome as evidenced by a recent study showing that only one third of all patients had a normal glucose tolerance test 3 months after the acute event [11]. These results are consistent with a 2002 prospective study by Norhammar and colleagues [12], who looked at outcomes in 181 consecutive patients admitted to the coronary care units of two hospitals in Sweden with acute myocardial infarction but no diagnosis of diabetes and a blood glucose concentration of less than $200 \mathrm{mg} / \mathrm{dL}(11.1 \mathrm{mmol} / \mathrm{L})$. Subjects underwent standardized oral glucose tolerance tests (75-g glucose load) at discharge and again 3 months later. Fifty-eight of $164(35 \%)$ and 58 of $144(40 \%)$ individuals had impaired glucose tolerance at discharge and after 3 months, respectively, whereas 51 of $164(31 \%)$ and 36 of $144(25 \%)$ had previously undiagnosed diabetes mellitus. 
It is well established that the short- and long-term prognosis of diabetic patients sustaining acute myocardial infarction is poor and is associated with increased mortality and morbidity [13]. A meta-analysis of 15 studies reported that hyperglycemia (glucose $>110 \mathrm{mg} / \mathrm{dL}$ ), with or without a prior history of diabetes, is associated with increases in in-hospital mortality and congestive heart failure in patients admitted for acute myocardial infarction [14].

The DIGAMI study demonstrated that the unfavorable long-term prognosis could be improved by insulin treatment [4], extending findings from previous reports [15-17]. In the DIGAMI study, 620 patients with acute myocardial infarction and hyperglycemia (with and without prior history of diabetes) were randomized to either intravenous (IV) insulinglucose infusion for at least 24 hours, followed by multidose subcutaneous insulin treatment for at least 3 months or to a control group, which received conventional treatment that generally included sulfonylurea therapy. The conventional treatment group was used as the control arm; therapy was left to the discretion of the treating physicians. Standard therapy for acute myocardial infarction was applied to all subjects. The baseline characteristics were similar between the two groups. The primary end point of the study was 3-month mortality; the secondary end point was 1-year mortality.

Results showed that the mortality at 1 year was significantly reduced in the infusion group $(19 \%)$ compared with the control group $(26 \%)$. The long-term results (3.4 years of follow-up) showed a persistent relative mortality reduction of $25 \%(P=0.011)$ in the insulin-treated group, which corresponds to an absolute mortality reduction of $11 \%$. Risk reduction was even more significant in patients who were considered "low risk": a group of 272 patients with no prior history of insulin therapy. In this group, there was a $58 \%$ reduction in mortality at discharge $(P<0.05)$, a $50 \%$ reduction at 12 months, and a $45 \%$ reduction at 3.4 years $(P=0.004)$.

\section{Impact of insulin therapy on nonglycemic parameters of metabolic control}

Insulin promotes glucose oxidation, which is beneficial in ischemic situations [18]. Increased levels of circulating free fatty acids (FFAs) are a common finding in a setting of myocardial ischemia [19]. FFA oxidation is detrimental to the myocardium because of an increased oxygen demand and to a direct inhibition of glucose oxidation [20]. In addition, increased FFA use during ischemia causes accumulation of FFA metabolites. These in turn are toxic to the myocardium and can provoke arrhythmia and exacerbate mechanical dysfunction [19]. Furthermore, the Paris Prospective Study reported FFAs as a predictor of sudden death [21], and the infusion of FFAs can induce ventricular fibrillation [19,22].

Proinflammatory cytokines are also likely involved in the morbidity and mortality of severe illness [23,24]. These cytokines appear to promote acute thrombosis, sepsis, heart failure, the cachexia of malignancy, and are 
involved in the pathogenesis of atherosclerosis. The regulation of these cytokines (and the acute phase proteins they modulate) is complex and not fully understood. However, one fact remains clear: exogenous insulin, when infused to normal or near-normal blood glucose levels, can inhibit some of these macrophage and monocyte products [25-27].

There are two other possible mechanisms through which insulin may independently improve outcomes in critically ill patients. First, it suppresses the growth factors involved in acute thrombosis, which may be important during acute myocardial infarction [28]. Second, it stimulates endothelial nitric oxide synthase, which results in the synthesis of nitric oxide and potentially results in vascular vasodilation [29].

\section{Impact of hyperglycemia and intravenous insulin infusion on cardiac surgery outcome}

The prevalence of diabetes among patients undergoing coronary artery bypass graft $(\mathrm{CABG})$ is as high as $28 \%$, and diabetes is an independent risk factor for CABG-related death [30]. In addition, postoperative hyperglycemia $(\geq 200 \mathrm{mg} / \mathrm{dL})$ is a predictor of infectious complications in patients undergoing coronary artery surgery [31] and is associated with surgical-site infection [32]. In a study of 1000 patients undergoing cardiothoracic surgery at a large university hospital setting, hyperglycemia in the first 48 postoperative hours was associated with a twofold higher rate of surgical-site infection compared with the normoglycemic group [32].

It has been shown that IV insulin during the postoperative period improves outcome. Zerr and colleagues [33] were able to demonstrate that use of IV insulin in the first 3 postoperative days significantly reduces morbidity in cardiac surgery patients, specifically in reducing deep sternal wound infection. This retrospective study involved 1585 patients with diabetes who underwent cardiac surgery between 1987 and 1993. The study looked at the rate of deep wound infection in patients treated before and after September 1991, when a protocol of postoperative continuous IV insulin to maintain a blood glucose level of less than $200 \mathrm{mg} / \mathrm{dL}$ was initiated. Patients treated with IV insulin infusion to maintain glucose levels below $200 \mathrm{mg} / \mathrm{dL}$ had a significantly lower incidence $(P<0.02)$ of deep wound infection, from $2.8 \%$ before the implementation of IV insulin therapy to $0.74 \%$ the third year after the implementation. In a subsequent publication, Furnary and colleagues [34] reported that continuous insulin infusion added an independently protective effect against death (odds ratio 0.50 , $P=.005)$ to the constellation of risk factors described in the Society of Thoracic Surgeons risk model [30]. They concluded that diabetes per se is not a true risk factor for death after CABG; rather, it is the underlying glycometabolic state of the myocardium that independently affects postoperative mortality. 


\section{Relationship between glycemic control and stroke outcome}

Observational studies suggest a correlation between blood glucose level, mortality and morbidity, and functional recovery in patients who have suffered a stroke. Capes and colleagues [35] performed a meta-analysis of 26 studies published between 1996 and 2000 and reported that in patients with no history of diabetes who had an ischemic stroke, even a moderately elevated glucose level $(>110 \mathrm{mg} / \mathrm{dL})$ is associated with a threefold higher risk of in-hospital or 30-day mortality and an increased risk of poor functional recovery compared with those with lower glucose levels. This metaanalysis was limited by several factors, including the use of pooled studies with different inclusion and exclusion criteria, definitions of hyperglycemia, and concomitant treatment; nevertheless, the strong association between admission hyperglycemia and poor prognosis suggests that glucose level is an important prognostic factor for morbidity and mortality after stroke.

Other studies suggest similar correlation between blood glucose level and mortality and morbidity in stroke patients [36,37]. These observational studies highlight the need for interventional controlled trials to investigate the impact of targeted glycemic control on the outcome of acute stroke both in patients with and without a known history of diabetes.

\section{Hyperglycemia and infection}

The association between hyperglycemia and infection has long been recognized [38,39]. Pomposelli and coworkers [38] found that early postoperative glucose control predicts nosocomial infection in diabetic patients. In this study, 97 patients with diabetes undergoing surgery were studied. On postoperative day 1, a single glucose level of more than $220 \mathrm{mg} / \mathrm{dL}$ was shown to be a sensitive predictor of nosocomial infection at a rate 2.7 times higher than in patients who had blood glucose levels below $220 \mathrm{mg} / \mathrm{dL}$. In a separate evaluation that excluded patients with minor urinary tract infection from the analysis, the rate of severe infection such as sepsis, pneumonia, and wound infection was increased to $5.7 \%$.

In 1499 patients studied between 1987 and 1994, Furnary and colleagues [6] found that hyperglycemia in the first 48 hours following surgery was an independent risk factor for deep sternal wound infection; patients with glucose levels above $200 \mathrm{mg} / \mathrm{dL}$ had a risk 2.2 times higher than those patients with glucose levels of less than $200 \mathrm{mg} / \mathrm{dL}$. Control of hyperglycemia with IV insulin in these patients resulted in a $66 \%$ reduction of this serious complication to a rate similar to that of nondiabetic subjects.

The association between hyperglycemia and increased infection may be attributed to impaired immune function. Phagocyte dysfunction appears to be the primary problem, including impaired adherence, chemotaxis, phago- 
cytosis, bacterial killing, and respiratory burst [40-50]. Other abnormalities of impaired immune function associated with hyperglycemia include nonenzymatic glycation of immunoglobulins and reduced T-lymphocyte populations [51]. The effect on peripheral lymphocytes associated with hyperglycemia is reversed when glucose levels are lowered [52].

\section{Good glycemic control reduces cost and length of hospital stay}

Although the association between hyperglycemia and adverse outcomes is well supported [53-56], prospective studies for using glucose reduction as a means of reducing length of hospitalization are few, and cost-effectiveness analyses are even fewer in number.

Several studies have shown a relationship between improved inpatient glucose control and decreased hospital length of stay [34,57-62]. Furnary and colleagues [62] showed that length of stay was increased by 1 day for each $50-\mathrm{mg} / \mathrm{dL}$ increase above $150 \mathrm{mg} / \mathrm{dL}$ in the mean glucose during the first 3 postoperative days $(P<0.001)$. An intensive IV insulin regimen was associated with $23 \%$ reduction in length of hospital stay. This resulted in a net savings of more than $\$ 680$ per patient.

In a nonrandomized, retrospective review, Levetan and associates [58] reported that diabetes team consultation resulted in a $56 \%$ shorter hospitalization than those patients with no consultation and proposed a cost saving of $\$ 2353$ per patients who were managed by the diabetes team. From the studies available, the cost benefit of improved glycemic control appears to be apparent; however, more rigorous analyses of cost effectiveness are warranted.

\section{The use of intravenous insulin in the hospital}

In the ICU, the data support the use of IV insulin both for critically ill patients (especially those who have had recent surgery) and for those with hyperglycemia during an acute myocardial infarction $[9,63]$. There is also general consensus that IV insulin should be used for metabolically unstable patients with widely fluctuating blood glucose levels, regardless of their location in the hospital $[9,63]$. However, IV insulin infusions are infrequently used on general medical and surgical wards. Typical "sliding scale" insulin is not effective in the manner that it is typically used [64], and yet it is part of our medical culture [65].

IV insulin infusions have been used to manage diabetes for more than 20 years and have a proven record of safety and efficacy [66]. An insulin drip has more predictable bioavailability, is easier and quicker to titrate, and is safer than subcutaneous insulin in patients who are not eating or who may be suddenly switched to no-eating status. 
There are a wide variety of insulin infusion protocols, but there are no studies in which algorithms are compared with one another. It is apparent that protocols have to be adapted to each specific hospital environment and that no one protocol would be likely to be effective at all hospitals. As an example, Appendix 1 presents the protocol used by the author at the University of Washington Medical Center in Seattle, Washington [67].

Several aspects of the IV insulin protocol need to be emphasized. First, there must be enough bedside glucose testing to ensure optimum patient safety. The ideal frequency of glucose testing has not been systematically studied. At the University of Washington, we generally initiate the protocol with hourly glucose measurements, at least until the blood glucose is stable [67]. Next, the protocol should include some mechanism for changing infusion rate if there have been significant alterations in blood glucose. Some of the most widely used protocols have this feature $[68,69]$.

Next, enough glucose needs to be provided to prevent both hypoglycemia and starvation ketosis. Again, the ideal amount has not been systematically studied, but most authors suggest 5 to $10 \mathrm{~g}$ each hour. If ketonuria occurs despite well-controlled blood glucose levels, the amount of glucose needs to be increased.

The University of Washington Medical Center is a 400-bed teaching hospital where numerous IV insulin infusion protocols have been used since 1991. Each service had their own protocol, but in July of 2002 the insulin infusion algorithms were all standardized to a protocol adapted from the Markovitz report (see Appendix 1) [68]. Instead of introducing the protocol throughout the entire hospital at the same time, each nursing unit was transitioned sequentially until they were comfortable with the new protocol. This took over a year to accomplish, but we found satisfaction from nurses and physicians to be high, and the protocol was found to be quite safe [70]. Compared with the old protocol, which resulted in over $40 \%$ of blood glucose values above $180 \mathrm{mg} / \mathrm{dL}$, we found only $16.7 \%$ of blood glucose levels greater than this value. Likewise, hypoglycemia was rare with the new protocol. Only 3\% had one blood glucose measurement below $40 \mathrm{mg} / \mathrm{dL}$, and $16 \%$ had one reading below $60 \mathrm{mg} / \mathrm{dL}$. On the old protocol, this compares to $14 \%$ and $30 \%$ below $40 \mathrm{mg} / \mathrm{dL}$ and $60 \mathrm{mg} / \mathrm{dL}$, respectively.

It is clear that for these types of protocols to work effectively, there must be an understanding of the general philosophies of insulin therapy among endocrinologists, cardiologists, surgeons, anesthesiologists, primary care physicians, nurses, and hospital pharmacists. Often a significant amount of education will be required for those not very familiar with insulin therapy.

According to the American College of Endocrinology position statement on inpatient diabetes and metabolic control [9], implementation of these protocols requires assessment of hospital systems for safety and quality of care; adjustments may be required for appropriate provision of diabetes care, 
including timely delivery of meal trays, point-of-care blood glucose testing, and the administration of diabetes medications. It is also recommended that nursing staff receive adequate and ongoing in-service training on the specialized needs of the inpatient with diabetes, especially with regard to insulin therapy. A team approach to implementation is also recommended. In addition to the physician, the team may include specialty staff such as a certified diabetes educator. Diabetes educators and nursing staff should collaborate in providing basic "survival skills" when needed to allow for a safe discharge. Finally, discharge planning should be initiated well in advance, exploring community resources and arranging for follow-up of diabetic issues.

\section{The use of subcutaneous insulin therapy in the hospital}

When addressing subcutaneous (SC) insulin therapy, it is important that the definitions of insulin therapy are appreciated. Differences between physiologic and nonphysiologic insulin replacement, basal and prandial insulin replacement, and the difference between a supplement and an adjustment need to be clear [66]. The insulin supplement (or "correction dose") needs to be differentiated from sliding scale insulin $[34,66]$.

Neutral protamine Hagedorn and regular insulin have been traditionally used for basal and prandial glucose coverage, respectively. We believe that the use of current insulin analogs minimizes hypoglycemia and increases flexibility [71]. It is important to appreciate that even though studies have not been performed, rapid-acting analogs (lispro or aspart) should theoretically be more effective and safer as correction dose insulin than regular insulin. This is because their more rapid onset and shorter duration of action makes them more predictable. By the same token, it is our opinion that, although using a pure basal insulin (eg, insulin glargine) to treat acute hyperglycemia will result in failure, glargine does provide a much more predictable basal delivery of insulin compared with neutral protamine Hagedorn or ultralente insulin.

Appendix 1 presents a sample SC order form that was developed and implemented at the University of Washington Medical Center to improve safety and efficacy of SC insulin [67]. Compared with the IV insulin infusion protocols, there is much less experience with these SC protocols. However, our protocol has resulted in several advantages, including some that were unexpected (Appendix 2). First, like all hospital protocols, having a uniform $\mathrm{SC}$ insulin algorithm results in standardization of therapy. Any problems can be rectified and there can be immediate quality improvements in the entire hospital system. Second, the protocol is designed to improve glucose control to better match the new guidelines [9]. Third, and perhaps most importantly, an SC insulin protocol that differentiates basal insulin replacement, prandial insulin replacement, and correction dose insulin can educate both younger and older physicians in current strategies for insulin 
use [71]. This last benefit was unexpected. Over the years there have been numerous attempts to better educate physicians about both the basics and the subtleties of insulin therapy, but surveys have reported that physicians do not have a good understanding of how to best use insulin, especially in the United States [72,73]. By using an SC protocol for hospitalized patients on a daily basis, physicians learn how to adjust insulin appropriately, including the best use of correction dose insulin. We believe that in relatively short amounts of time, medical and surgical residents of all specialties could become quite knowledgeable about the use of SC insulin by simply working with the protocol for their hospitalized patients. Only prospective trials will be able to confirm these beliefs.

\section{Summary}

The evidence continues to strengthen our understanding that improved glycemic control with the use of insulin therapy may significantly improve morbidity and mortality in hospitalized patients with hyperglycemia, with or without a previous diagnosis of diabetes. However, many questions remain concerning the impact and relative contributions of blood glucose and insulin per se. Nevertheless, the publication of numerous and consistent studies have made it clear that the topic of glycemic management in the hospital requires a larger priority among clinicians caring for these patients.

The recently published guidelines by the American Association of Clinical Endocrinologists are the first formal recommendations on this topic [9], but national guidelines for blood glucose levels cannot take into account all of the different challenges facing different hospitals. This suggests that each institution will require individualization of protocols even though the ultimate metabolic goals are identical. Furthermore, it is not realistic to expect those unfamiliar with diabetes therapy to appreciate all of the nuances and vagaries of insulin treatment. Like any medical treatment, a significant amount of time will need to be invested by the providers involved with the care of these patients before a mastery of the therapy can be achieved. Nevertheless, because the rewards to our patients can be significant, we need to strive to improve the systems where we work. Individual clinicians with vast experience in diabetes care cannot be successful for the inpatient with diabetes unless the hospital has systems in place to effectively and efficiently facilitate the management of the metabolic needs of this population.

The main challenge now is the safe and effective implementation of these guidelines in both small and large hospitals given the limited level of resources available in today's medical environment. Therefore, our single most important recommendation is to ensure that all clinicians involved in the management of these patients are in agreement about general philosophies of diabetes management. We would recommend that there are "champions" for each discipline: endocrinology, cardiology, anesthesiology, surgery, nursing, 
and pharmacy, all of which have developed hospital-specific guidelines for glycemic management. These recommendations can be slowly adapted, one unit at a time, until the entire hospital has transitioned to a more "diabetesfriendly" environment. The ultimate goal of well-controlled glycemia with minimal hypoglycemia should be possible for most hospitals, and we hope this review will assist clinicians in achieving this objective.

We await additional outcome research with carefully controlled studies to confirm the value of these recommendations at different levels of glycemic control. We believe that we can already state with confidence that the preliminary evidence shows that, like outpatient diabetes management, metabolic control matters during acute illness.

\section{Appendix 1. Example of a standardized intravenous insulin infusion}

\section{General guidelines}

- Goal blood glucose level: usually 80 to $180 \mathrm{mg} / \mathrm{dL}$ (80 to $110 \mathrm{mg} / \mathrm{dL}$ for the ICU)

- Standard drip: $100 \mathrm{U} / 100 \mathrm{~mL} 0.9 \% \mathrm{NaCl}$ by way of an infusion device (1 U/1 cc)

- Surgical patients who have received an oral diabetes medication within 24 hours should start when blood glucose level is above $120 \mathrm{mg} / \mathrm{dL}$. All other patients can start when blood glucose level is $70 \mathrm{mg} / \mathrm{dL}$ or more

- Insulin infusions should be discontinued when a patient is eating and has received first dose of subcutaneous insulin

\section{Intravenous fluids}

- Most patients will need 5 to $10 \mathrm{~g}$ of glucose per hour

- $\mathrm{D}_{5} \mathrm{~W}$ or $\mathrm{D}_{5} \mathrm{~W} 1 / 2 \mathrm{NS}$ at 100 to $200 \mathrm{~mL}$ per hour or equivalent (total parenteral nutrition, enteral feeds, and so forth)

\section{Initiating the infusion}

- Algorithm 1: start here for most patients.

- Algorithm 2: for patients not controlled with algorithm 1, or start here if status post (s/p) CABG, s/p solid organ transplant or islet cell transplant, receiving glucocorticoids, or patient with diabetes receiving more than $80 \mathrm{U} / \mathrm{d}$ of insulin as an outpatient

- Algorithm 3: for patients not controlled with algorithm 2; no patients start here without authorization from the endocrine service

- Algorithm 4: for patients not controlled with algorithm 3; no patients start here 
Patients not controlled with the above algorithms require an endocrine consult.

\begin{tabular}{|c|c|c|c|c|c|c|c|}
\hline \multicolumn{2}{|c|}{ Algorithm 1} & \multicolumn{2}{|l|}{ Algorithm 2} & \multicolumn{2}{|l|}{ Algorithm 3} & \multicolumn{2}{|l|}{ Algorithm 4} \\
\hline BG & Units/hour & BG & Units/hour & BG & Units/hour & BG & Units/hour \\
\hline$<70$ & Off & $<70$ & Off & $<70$ & Off & $<70$ & Off \\
\hline 70-109 & 0.2 & 70-109 & 0.5 & 70-109 & 1 & 70-109 & 1.5 \\
\hline $110-119$ & 0.5 & $110-119$ & 1 & $110-119$ & 2 & $110-119$ & 3 \\
\hline $120-149$ & 1 & $120-149$ & 1.5 & $120-149$ & 3 & $120-149$ & 5 \\
\hline $150-179$ & 1.5 & $150-179$ & 2 & $150-179$ & 4 & $150-179$ & 7 \\
\hline $180-209$ & 2 & $180-209$ & 3 & $180-209$ & 5 & $180-209$ & 9 \\
\hline $210-239$ & 2 & $210-239$ & 4 & $210-239$ & 6 & $210-239$ & 12 \\
\hline $240-269$ & 3 & $240-269$ & 5 & $240-269$ & 8 & $240-269$ & 16 \\
\hline $270-299$ & 3 & $270-299$ & 6 & $270-299$ & 10 & $270-299$ & 20 \\
\hline $300-329$ & 4 & $300-329$ & 7 & $300-329$ & 12 & $300-329$ & 24 \\
\hline $330-359$ & 4 & $330-359$ & 8 & $330-359$ & 14 & $>330$ & 28 \\
\hline$>360$ & 6 & $>360$ & 12 & $>360$ & 16 & - & - \\
\hline
\end{tabular}

$<60=$ hypoglycemia (see below for treatment).

Abbreviation: BG, blood glucose.

\section{Moving from algorithm to algorithm}

- Moving up: an algorithm failure is defined as a blood glucose level outside the goal range (see above goal), and the blood glucose does not change by at least $60 \mathrm{mg} / \mathrm{dL}$ within 1 hour

- Moving down: when blood glucose level is less than $70 \mathrm{mg} / \mathrm{dL} \times 2$

\section{Patient monitoring}

- Goal blood glucose level: 80 to $180 \mathrm{mg} / \mathrm{dL}$

- Check capillary blood glucose level every hour until it is within goal range for 4 hours, then decrease to every 2 hours for 4 hours; if it remains stable, decrease to every 4 hours

- Hourly monitoring may be indicated for critically ill patients even if they have stable blood glucose

Treatment of hypoglycemia (blood glucose level $<60 \mathrm{mg} / \mathrm{dL}$ )

- Discontinue insulin drip AND

- Give $\mathrm{D}_{50} \mathrm{~W}$ IV (patient awake, $25 \mathrm{~mL}$ [1/2 amp]; patient not awake, 50 $\mathrm{mL}$ [1 amp])

- Recheck blood glucose level every 20 minutes and repeat $25 \mathrm{~mL}$ of $\mathrm{D}_{50} \mathrm{~W}$ IV if it is below $60 \mathrm{mg} / \mathrm{dL}$; restart drip once blood glucose is above $70 \mathrm{mg} / \mathrm{dL} \times 2$ checks; restart drip with lower algorithm (see Moving Down) 
When to notify the physician

- If there is any blood glucose change greater than $100 \mathrm{mg} / \mathrm{dL}$ in 1 hour

- If blood glucose level is above $360 \mathrm{mg} / \mathrm{dL}$

- For hypoglycemia that has not resolved within 20 minutes of administering $50 \mathrm{~mL}$ of $\mathrm{D}_{50} \mathrm{~W}$ IV and discontinuing the insulin drip

Adapted from Trence DL, Kelly JL, Hirsch IB. The rationale and management of hyperglycemia for in-patients with cardiovascular disease: time for change. J Clin Endocrinol Metab 2003;88:2430-7; with permission.

\section{Appendix 2. Example of standardized subcutaneous insulin orders}

Blood glucose monitoring

- Before meals and at bedtime

- _ hours after meals

- 2:00 to 3:00 AM

\begin{tabular}{lllll}
\hline Insulin & & & & \\
orders & Breakfast & Lunch & Dinner & Bedtime \\
\hline Prandial & Give_units of: & Give_units of: & Give_units of: & - \\
& Lispro & Lispro & Lispro & \\
& Aspart & Aspart & Aspart & \\
& Regular & Regular & Regular & \\
Basal & Give_units of: & - & Give_units of: & Give_ units of: \\
& NPH & & NPH & NPH \\
& Lente & & Lente & Lente \\
& Ultralente & & Ultralente & Ultralente \\
& Glargine & & Glargine & Glargine \\
\hline
\end{tabular}

Goal premeal blood glucose: $80-150 \mathrm{mg} / \mathrm{dL}$.

Suggested lag times for prandial insulin:

- Aspart/Lispro: 0 to 15 minutes before eating

- Regular: 30 minutes before eating

If blood glucose is $<60 \mathrm{mg} / \mathrm{dL}$ :

A. If patient can take orally, give $15 \mathrm{~g}$ of fast-acting carbohydrate (4-oz fruit juice/nondiet soda, 8-oz nonfat milk, or 3 to 4 glucose tablets)

B. If patient cannot take orally, give $25 \mathrm{~mL}$ of D50 as IV push

C. Check finger capillary glucose every 15 minutes and repeat above if blood glucose is $<80 \mathrm{mg} / \mathrm{dL}$ 
Premeal "correction dose" algorithm for hyperglycemia to be administered in addition to scheduled insulin dose to correct premeal hyperglycemia.

- Lispro

- Aspart

\begin{tabular}{lll}
\hline Algorithm & Premeal BG & Additional insulin (units) \\
\hline Low-dose $^{\mathrm{a}}$ & $150-199$ & 1 \\
& $200-249$ & 2 \\
& $250-299$ & 3 \\
& $300-349$ & 4 \\
& $>349$ & 5 \\
Medium-dose $^{\mathrm{b}}$ & $150-199$ & 1 \\
& $200-249$ & 3 \\
& $250-299$ & 5 \\
& $300-349$ & 7 \\
High-dose $^{\mathrm{c}}$ & $>349$ & 2 \\
& $150-199$ & 4 \\
& $200-249$ & 7 \\
Individualized & $250-299$ & 10 \\
& $300-349$ & \\
& $>349$ & \\
& $150-199$ & \\
& $200-249$ & \\
& $250-299$ & \\
& $300-349$ & \\
& $>349$ & \\
\end{tabular}

Abbreviation: $\mathrm{BG}$, blood glucose.

${ }^{\text {a }}$ For patients requiring $\leq 40 \mathrm{U}$ of insulin per day.

${ }^{\mathrm{b}}$ For patients requiring $40-80 \mathrm{U}$ of insulin per day.

${ }^{\mathrm{c}}$ For patients requiring $>80 \mathrm{U}$ of insulin per day.

\section{General insulin dosing recommendations}

\section{Patients with type 1 diabetes}

This patient must have insulin to prevent ketosis. Even if the patient is not eating, he/she will need at least basal insulin $(\mathrm{NPH} /$ lente/ultralente/ glargine) to prevent ketosis.

1. When admitting a patient with type 1 diabetes, continue the basal insulin that the patient was taking at home at the same dose. If the patient will not be eating, use an insulin drip rather than subcutaneous insulin. The prandial insulin (regular/lispro/aspart) may require adjustment depending on the patient's situation. If the patient is eating much less, the prandial insulin will need to be reduced. Many hospitalized patients are under significant metabolic stress (infection, glucocorticoids, and so forth) and may require larger doses of prandial insulin despite eating less.

2. If a patient is newly diagnosed, the usual daily insulin requirement is 0.5 to $0.7 \mathrm{U} / \mathrm{kg} / \mathrm{d}$. Half should be given as basal insulin and the remainder as prandial insulin. 


\section{Patients with type 2 diabetes}

1. If patient is using insulin at home, continue the outpatient regimen and adjust as needed.

2. If patient has not been using insulin previously, the usual total daily insulin requirement is 0.4 to $1.0 \mathrm{U} / \mathrm{kg} / \mathrm{d}$.

3. Individual insulin doses vary widely and adjustments should be made based on the bedside and laboratory glucose levels.

Note: Individual insulin doses vary widely, and adjustments should be based on bedside and laboratory glucose levels.

Adapted from Trence DL, Kelly JL, Hirsch IB. The rationale and management of hyperglycemia for in-patients with cardiovascular disease: time for change. J Clin Endocrinol Metab 2003;88:2430-87; with permission.

\section{References}

[1] Centers for Disease Control and Prevention (CDC). Diabetes fact sheet. Available at: http:// diabetes.org/diabetes-statistics/national-diabetes-fact-sheet.jsp. Accessed February 20, 2004.

[2] Narayan KMV, Boyle JP, Thompson TJ, et al. Lifetime risk for diabetes mellitus in the United States. JAMA 2003;290:1884-90.

[3] Hogan P, Dall T, Nikolov P. American Diabetes Association. Economic costs of diabetes in the US in 2002. Diabetes Care 2003;26:917-32.

[4] Malmberg K, Norhammar A, Wedel H, et al. Glycometabolic state at admission: important risk marker of mortality in conventionally treated patients with diabetes mellitus and acute myocardial infarction: long-term results from the Diabetes and Insulin-Glucose Infusion in Acute Myocardial Infarction (DIGAMI) study. Circulation 1999;99:2626-32.

[5] Van den Berghe G, Wouters P, Weekers F, et al. Intensive insulin therapy in the critically ill patients. N Engl J Med 2001;345:1359-67.

[6] Furnary AP, Zerr KJ, Grunkemeier GL, et al. Continuous intravenous insulin infusion reduces the incidence of deep sternal wound infection in diabetic patients after cardiac surgical procedures. Ann Thorac Surg 1999;67:352-60 [discussion: 360-2].

[7] Lazar HL, Chipkin SR, Fitzgerald CA, et al. Tight glycemic control in diabetic coronary artery bypass graft patients improves perioperative outcomes and decreases recurrent ischemic events. Circulation 2004;109:1497-502.

[8] Centers for Disease Control and Prevention. Diabetes Public Health Resource. Available at: http://www.cdc.gov/diabetes/statistics. Accessed February 20, 2004.

[9] American College of Endocrinology. Position statement on inpatient diabetes and metabolic control. Endocr Pract 2004;10:77-82.

[10] Webster MW, Scott RS. What cardiologists need to know about diabetes. Lancet 1997; 350(Suppl): 123-8.

[11] Tenerz A, Norhammar A, Silveira A, et al. Diabetes, insulin resistance, and the metabolic syndrome in patients with acute myocardial infarction without previously known diabetes. Diabetes Care 2003;26:2770-6.

[12] Norhammar A, Tenerz A, Nilsson G, et al. Glucose metabolism in patients with acute myocardial infarction and no previous diagnosis of diabetes mellitus: a prospective study. Lancet 2002;359:2140-4.

[13] Abbott R, Kannel W, Wilson P. The impact of diabetes on survival following myocardial infarction in men and women. JAMA 1988;260:3456-60. 
[14] Capes SE, Hunt D, Malmberg K, et al. Stress hyperglycemia and increased risk of death after myocardial infarction in patients with and without diabetes: a systematic overview. Lancet 2000;355:773-8.

[15] Malmberg K, Ryden L, Efendic S, et al. A randomized trial of insulin-glucose infusion followed by subcutaneous insulin treatment in diabetic patients with acute myocardial infarction: effects on one year mortality. J Am Coll Cardiol 1995;26:57-65.

[16] Malmberg K, Ryden L, Hamsten A, et al. Effects of insulin treatment on cause-specific oneyear mortality and morbidity in diabetic patients with acute myocardial infarction. Eur Heart J 1996;17:1337-44.

[17] Malmberg K. Prospective randomized study of intensive insulin treatment on long-term survival after acute myocardial infarction in patients with diabetes mellitus. BMJ 1997;314: $1512-5$.

[18] Perdomo G, Commerford SR, Richard A-MT, et al. Increased beta-oxidation in muscle cells enhances insulin-stimulated glucose metabolism and protects against fatty acid induced insulin resistance despite intramyocellular lipid accumulation. J Biol Chem 2004;279(26): $27177-86$.

[19] Oliver MF, Opie LH. Effects of glucose and fatty acids on myocardial ischaemia and arrhythmias. Lancet 1994;343:155-8.

[20] Lopaschuk GD, Wambolt RB, Barr RL. An imbalance between glycolysis and glucose oxidation is a possible explanation for the detrimental effects of high levels of fatty acids during aerobic reperfusion of ischemic hearts. J Pharmacol Exp Ther 1993;264:135-44.

[21] Jouven X, Charles MA, Desnos M, et al. Circulating nonesterified fatty acid level as a predictive risk factor for sudden death in the population. Circulation 2001;104:756-61.

[22] Hirsch IB. Impact of insulin therapy on non-glycemic effects during acute illness. Endo Pract 2004;10(Suppl 2):63-70.

[23] Gabay C, Kushner I. Acute phase proteins and other systemic responses to inflammation. N Engl J Med 1999;340:448-54.

[24] Zeidler C, Kanz L, Hurkuck KL. In vivo effects of interleukin-6 on thrombopoiesis in healthy and irradiated primates. Blood 1992;80:2740-5.

[25] Dandona P, Aljada A, Bandyopadhyay A. The potential therapeutic role of insulin in acute myocardial infarction in patients admitted to intensive care and in those with unspecified hyperglycemia. Diabetes Care 2003;26:516-9.

[26] Dandona P, Aljzcz Z, Mohanty P, et al. Insulin inhibits intranuclear factor $\kappa \beta$ and stimulates $\kappa \beta$ in mononuclear cells in obese subjects: evidence for an anti-inflammatory effect. J Clin Endocrinol Metab 2001;86:3257-65.

[27] Aljada A, Ghanim H, Saadeh R, et al. Insulin inhibits NF- $\kappa \beta$ and MCP-1 expression in human aortic endothelial cells. J Clin Endocrinol Metab 2001;86:450-3.

[28] Ghanim H, Mohanty P, Aljada A, et al. Insulin reduces the pro-inflammatory transcription factor, activation protein-1 (AP-1), in mononuclear cells (MNC) and plasma matrix metalloproteinase-9 (MMP-9) concentration. Diabetes 2001;50(Suppl 1):A408.

[29] Steinberg HO, Brechtel G, Johnson N, et al. Insulin-mediated skeletal muscle vasodilation is nitric-oxide dependent. A novel action of insulin to increase nitric oxide release. J Clin Invest 1994;94:1172-9.

[30] Edwards FH, Grover FL, Shroyer AL, et al. The Society of Thoracic Surgeons, National Cardiac Surgery Database: current risk assessment. Ann Thorac Surg 1997;63:903-8.

[31] Golden SH, Pear-Vigilance C, Kao WH, et al. Preoperative glycemic control and the risk of infectious complications in a cohort of adult with diabetes. Diabetes Care 1999;22:1408-14.

[32] Latham R, Lancaster AD, Covington JF, et al. The association of diabetes and glucose control with surgical site infection among cardiothoracic surgery patients. Infect Control Hosp Epidemil 2001;22:607-12.

[33] Zerr KJ, Furnary AP, Grunkemeier GL, et al. Glucose control lowers the risk of wound infection in diabetics after open heart operations. Ann Thorac Surg 1997;63:356-61. 
[34] Furnary AP, Gao G, Grunkemeier GL, et al. Continuous insulin infusion reduces mortality in patients with diabetes undergoing coronary artery bypass grafting. J Thorac Cardiovasc Surg 2003;125:1007-21.

[35] Capes SE, Hunt D, Malmberg K, et al. Stress hyperglycemia and prognosis of stroke in non diabetic and diabetic patients: a systematic overview. Stroke 2001;32:2426-32.

[36] Williams LS, Rotich J, Qi R, et al. Effects of admission hyperglycemia on mortality and costs in acute ischemic stroke. Neurology 2002;59:67-71.

[37] Pulsinelli WA, Levy DE, Sigsbee B, et al. Increased damage after ischemic stroke in patients with hyperglycemia with or without established diabetes mellitus. Am J Med 1983; 74:540-4.

[38] Pomposelli JJ, Baxter JK III, Babineau TJ, et al. Early postoperative glucose control predicts nosocomial infection rate in diabetic patients. J Parenter Enteral Nutr 1998;22:77-81.

[39] Golden SH, Peart-Vigilance C, Kao WH, et al. Perioperative glycemic control and the risk of infectious complications in a cohort of adults with diabetes. Diabetes Care 1999;22: $1408-14$.

[40] Joshi N, Caputo G, Weitekamp M, et al. Infections in patients with diabetes mellitus. N Engl J Med 1999;341:1906-12.

[41] Wheat L. Infection and diabetes mellitus. Diabetes Care 1980;3:187-97.

[42] Mowat A, Baum J. Chemotaxis of polymorphonuclear leukocytes from patients with diabetes mellitus. N Engl J Med 1971;284:621-7.

[43] Bagdade J, Root R, Bulger R. Impaired leukocyte function in patients with poorly controlled diabetes. Diabetes 1974;23:9-15.

[44] Bagdade JD, Stewart M, Walters E. Impaired granulocyte adherence. A reversible defect in host defense in patients with poorly controlled diabetes. Diabetes 1978;27:677-81.

[45] van Oss CJ, Border JR. Influence of intermittent hyperglycemic glucose levels on the phagocytosis of microorganisms by human granulocytes in vitro. Immunol Commun 1978;7: $669-76$.

[46] Davidson N, Sowden J, Fletcher J. Defective phagocytosis in insulin-controlled diabetics: evidence for a reaction between glucose and opsonizing proteins. J Clin Pathol 1984;37: $783-6$.

[47] Alexiewicz J, Kumar D, Smogorzewski M, et al. Polymorphonuclear leukocytes in noninsulin-dependent diabetes mellitus: abnormalities in metabolism and function. Ann Intern Med 1995;123:919-24.

[48] Leibovici L, Yehezkelli Y, Porter A, et al. Influence of diabetes mellitus and glycemic control on the characteristics and outcome of common infections. Diabet Med 1996;13:457-63.

[49] Kwoun M, Ling P, Lydon E, et al. Immunologic effects of acute hyperglycemia in nondiabetic rats. J Parenter Enteral Nutr 1997;21:91-5.

[50] McManus L, Bloodworth R, Prihoda T, et al. Agonist-dependent failure of neutrophil function in diabetes correlates with extent of hyperglycemia. J Leukoc Biol 2001;70: 395-404.

[51] Black CT, Hennessey PJ, Andrassy RJ. Short-term hyperglycemia depresses immunity through nonenzymatic glycosylation of circulating immunoglobulin. J Trauma 1990;30: 830-2 [discussion: 832-3].

[52] Bouter KP, Meyling FH, Hoekstra JB, et al. Influence of blood glucose levels on peripheral lymphocytes in patients with diabetes mellitus. Diabetes Res 1992;19:77-80.

[53] Selby JV, Ray GT, Zhang D, et al. Excess costs of medical care for patients with diabetes in a managed care population. Diabetes Care 1997;20:1396-402.

[54] Weir CJ, Murray GD, Dyker AG, et al. Is hyperglycaemia an independent predictor of poor outcome after acute stroke? Results of a long term follow up study. BMJ 1997;314:1303-6.

[55] Wahab NN, Cowden EA, Pearce NJ, et al. ICONS Investigators. Is blood glucose an independent predictor of mortality in acute myocardial infarction in the thrombolytic era? J Am Coll Cardiol 2002;40:1748-54. 
[56] Umpierrez GE, Isaacs SD, Bazargan N, et al. Hyperglycemia: an independent marker of in-hospital mortality in patients with undiagnosed diabetes. J Clin Endocrinol Metab 2002; 87:978-82.

[57] Koproski J, Pretto Z, Poretsky L. Effects of an intervention by a diabetes team in hospitalized patients with diabetes. Diabetes Care 1997;20:1553-5.

[58] Levetan CS, Salas JR, Wilets IF, et al. Impact of endocrine and diabetes team consultation on hospital length of stay for patients with diabetes. Am J Med 1995;99:22-8.

[59] Levetan CS, Passaro MD, Jablonski KA, et al. Effect of physician specialty on outcomes in diabetic ketoacidosis. Diabetes Care 1999;22:1790-5.

[60] Almbrand B, Johannesson M, Sjostrand B, et al. Cost-effectiveness of intense insulin treatment after acute myocardial infarction in patients with diabetes mellitus; results from the DIGAMI study. Eur Heart J 2000;21:733-9.

[61] Zhan C, Miller MR. Excess length of stay, charges, and mortality attributable to medical injuries during hospitalization. JAMA 2003;290:1868-74.

[62] Furnary AP, Chaugle H, Zerr KJ, et al. Postoperative hyperglycemia prolongs length of stay in diabetic CABG patients. Circulation 2000;102:II-556.

[63] Clement S, Braithwait SS, Magee MF, et al. Management of diabetes and hyperglycemia in hospitals. Diabetes Care 2004;27:553-91.

[64] Queale WS, Seidler AJ, Brancati FL. Glycemic control and sliding scale insulin use in medical inpatients with diabetes mellitus. Arch Intern Med 1997;157:545-52.

[65] Hirsch IB, Farkas-Hirsch R. Sliding scale or sliding scare: it's all sliding nonsense. Diabetes Spectrum 2001;14:79-81.

[66] Hirsch IB, Paauw DS, Brunzell J. Inpatient management of adults with diabetes. Diabetes Care 1995;18:870-8.

[67] Trence DL, Kelly JL, Hirsch IB. The rationale and management of hyperglycemia for inpatients with cardiovascular disease: time for change. J Clin Endocrinol Metab 2003;88: 2430-87.

[68] Markovitz L, Wiechmann R, Harris N, et al. Description and evaluation of a glycemic management protocol for diabetic patients undergoing heart surgery. Endocr Pract 2002;8: $10-8$.

[69] Albert Starr Academic Center for Cardiac Surgery. Star Wood Research. Available at: http://www.starrwood.com/research/insulin.html. Accessed March 14, 2004.

[70] Ku SY, Sayre CA, Hirsch IB, et al. New insulin infusion protocol improves blood control in hospitalized patients without increasing hypoglycemia. Jt Comm J Qual Improv, in press.

[71] Dewitt DE, Hirsch IB. Outpatient therapy for type 1 and type 2 diabetes: scientific review. JAMA 2003;289:2254-64.

[72] Tabak AG, Tamas G, Zgibor J, et al. Targets and reality: a comparison of healthcare indicators in the US (Pittsburgh Epidemiology of Diabetes Complications Study) and Hungary (DiabCare Hungary). Diabetes Care 2000;23:1284-9.

[73] Haywood RA, Manning WG, Kaplan SH, et al. Starting insulin therapy in patients with type 2 diabetes: effectiveness, complications, and resource utilization. JAMA 1997;278:1663-9. 